\title{
Artículos
}

\section{De la antropología demográfica a la arqueología demográfica: el estudio de las poblaciones extintas}

\section{From demographic anthropology to demographic archeology: The study of extinct populations}

\author{
Virginia Arieta Baizabal*
}

\section{Resumen}

Hay una tendencia actual a definir a la antropología demográfica como una propuesta nacida desde la antropología física. No obstante, este enfoque teórico-metodológico se fundamenta, desde sus inicios, en la profunda relación histórica entre la demografia y la antropología - esta última concebida desde una perspectiva holisti$c a$, donde necesariamente convergen los modelos teóricos, métodos y técnicas de todas las ramas antropológicas-. Este artículo muestra el aditamento de elementos al método de la denominada arqueología demográfica, para la obtención y análisis de información con la finalidad de inferir la estructura demográfica de poblaciones extintas a través del cálculo de habitantes por asentamiento y vivienda mediante nuevas tecnologías para el análisis espacial. Recurrimos a un estudio de caso en torno al sitio olmeca de San Lorenzo, con la finalidad de exponer el potencial del enfoque y mostrar que las investigaciones desde este campo, concebido como parte integral de la antropología demográfica, tienen que desarrollarse necesariamente desde la intradisciplina, interdisciplina y transdisciplina, con la finalidad de contribuir a la comprensión de las variadas y complejas estructuras de las poblaciones humanas del pasado.

Palabras clave: estimaciones poblacionales; antropología demográfica; arqueología demográfica; densidad poblacional.

\footnotetext{
Abstract

Nowadays, there is common perception that conceptualizes demographic anthropology as something born from physical anthropology. Nonetheless, this theoretical and

*Universidad Veracruzana, Instituto de Antropología. Dirección: Av. Xalapa 310, CP 91130, Xalapa, Veracruz, México. Correo: varieta@uv.mx ORCID: https://orcid.org/0000-00020932-6272
} 
methodological approach is based, since its inception, in the deeply rooted historical relation between demographics and anthropology - the latter understood in a holistic perspective, in which theories, methods and techniques from every anthropological discipline converge. The purpose of this paper is to advance a methodological reformulation of the so-called demographic archaeology, in order to be able to infer demographic structures from extinct populations through calculations of the number of inhabitants per household. We will turn to a case study of the Olmec site of San Lorenzo in order to expose the potential of the approach and show that research in this field, conceived as an integral part of a broader demographic anthropology, is necessarily developed through interdisciplinary, intradisciplinary and transdisciplinary approaches, applying new technologies with the ultimate goal of contributing to the comprehension of the varied and complex dynamics of human populations.

Keywords: population estimates; demographic anthropology; population density.

\section{Introducción}

El interés por conocer cuántos somos, por qué una población crece, disminuye o se distribuye de tal forma, por qué hay sociedades que desaparecen, por qué migramos o cuáles son las explicaciones e implicaciones de las variadas y complejas estructuras poblacionales, han sido y siempre serán cuestionamientos de reflexión histórica. Quizá ésta sea la razón del porqué en los últimos años han adquirido énfasis los estudios antropológicos con interés demográfico en torno a los factores dinámicos de las poblaciones prehispánicas, donde se puede obtener importante y valiosa información sobre edad, sexo, mortalidad, migración y fecundidad. Por la naturaleza de los temas, la mayoría de tales estudios han sido abordados mediante el estudio paleodemográfico; es decir, llevados a cabo por antropólogos físicos a través del análisis de material óseo. No obstante, desde nuestro punto de vista, un vínculo tan complejo como aquel entre los factores demográficos, culturales y medioambientales en la época antigua no debe de abordarse desde una sola perspectiva antropológica. Si de ello dependiera, muchos sitios de Mesoamérica no serían candidatos a la investigación del importante tema sobre la dinámica poblacional debido a la ausencia de entierros humanos, sobre todo los ubicados en las regiones húmedas donde los factores medioambientales no favorecen su preservación.

Consideramos que el enfoque teórico-metodológico de la antropología demográfica conlleva más profundidad. Por una parte, tal y como el sustantivo lo indica, tiene que entenderse desde un sentido holístico e intradisciplinario donde se incluyan otros campos de la antropología; por otra, como 
señala el adjetivo "demográfica", debe comprenderse desde la interdisciplina, e incluso desde la transdisciplina. Sobre todo cuando, dentro de las disciplinas antropológicas, la arqueología ha desarrollado una perspectiva sobre estudios demográficos de poblaciones antiguas que, aunque no es muy tratada, se fundamenta en una historia de casi cincuenta años. Hacemos referencia a la propuesta por Fekri Hassan en su publicación de 1978, denominada arqueología demográfica, tema central del presente artículo y en la que profundizaremos más adelante.

Antes, es indispensable una breve exposición de los antecedentes del vínculo entre la antropología y la demografía para los objetivos de este artículo, que son: 1) posicionar a la antropología demográfica como un enfoque que surge desde una perspectiva antropológica amplia y holística, donde necesariamente convergen las diversas perspectivas (arqueología demográfica surgida desde la arqueología y la paleodemografía como parte de la antropología física);2) añadir elementos a la metodología de la arqueología demográfica propuesta a finales de la década de los setenta, que incluyan la intradisciplina antropológica a través de la etnoarqueología, la interdisciplina mediante la arqueometría y la aplicación de nuevas tecnologías para la obtención y el análisis de la información (tal es el caso de las excavaciones con pruebas de barreno); y 3) mostrar los alcances y limitaciones del modelo propuesto desde la arqueología, que tiene como finalidad el estudio de la estructura poblacional a través de la cultura material, como alternativa a la ausencia de restos óseos, por medio de un estudio de caso. Para los fines de este tercer punto, presentamos el estudio sobre la implementación del enfoque en el sitio olmeca de San Lorenzo, Veracruz.

La meta de este artículo se suscribe en el interés por contribuir a la construcción de una propuesta epistemológica y metodológica del enfoque intradisciplinario, interdisciplinario y transdisciplinario denominado arqueología demográfica, que surge como una herramienta para el estudio de las estructuras poblacionales extintas.

\section{De la demografía antropológica a la antropología demográfica}

El presente artículo se centra en la documentación en torno al surgimiento y el desarrollo de los estudios demográficos en la arqueología, fundamentados en los planteamientos de una perspectiva más amplia y compleja, denominada antropología demográfica. No podemos adentrarnos en el enfoque de la arqueología demográfica, ni sustentarlo como parte de un enfoque mayor, sin hacer un breve recorrido general por la historia de los estudios de la población. 
Desde milenios atrás hay una gran inquietud por conocer y explicar todo lo relacionado con la población; las preguntas con las que inicia este texto son tan sólo una mínima parte. Marshall Sahlins, en el célebre libro Economía de la Edad de Piedra, menciona que las sociedades cazadoras-recolectoras del Paleolítico eran capaces de alcanzar la opulencia y satisfacer sus necesidades basándose en los recursos provenientes del medio ambiente; demoliendo de esta forma la visión de los evolucionistas lineales de la época (Sahlins, 1974, p. 24). Aunado a lo anterior, Marvin Harris, en su libro Caníbales y reyes, intenta explicar cómo pudo haber sido la vida en el pasado, con base en las prácticas actuales de grupos de cazadores-recolectores. Los antiguos pobladores, aparte de contar con alimentos que les proporcionaban una dieta saludable y nutritiva, sólo tenían que invertir un máximo de tres a cuatro horas diarias para satisfacer sus necesidades básicas (Harris, 1986, p. VII). Además, contaban con métodos para regular el crecimiento y la densidad poblacional; tal es el caso del aborto, el infanticidio y la prolongación del periodo de lactancia (Harris, 1986, p. 18). Desafortunadamente, contamos con muy poca o ninguna información de estudios demográficos sobre época tan temprana. Es sabido que Thomas Malthus es considerado el padre de la demografía moderna por su obra Essay on the principle of population, de 1798 , y por ser el primero en profundizar y desarrollar un modelo poblacional en el que intervienen varios factores, en aplicar el análisis estadístico a los censos de población, y en comparar datos de diversas regiones del mundo.

Hoy en día, la demografía es una disciplina consolidada que estudia todos los procesos que condicionan la formación, conservación y desaparición de las poblaciones a través de los indicadores de natalidad, mortalidad, nupcialidad, migración y fecundidad, entre otros; las variadas combinaciones de estos fenómenos en dimensiones numéricas; y las explicaciones sobre la composición de la población. Dichos objetivos de estudio son sumamente complejos para que la demografía actúe de manera independiente; es por ello por lo que en el presente existen diversas disciplinas científicas que trabajan junto a ella con la finalidad de profundizar en el estudio de la estructura de la población humana y la interrelación de sus factores, teniendo como propósito esencial el análisis de las múltiples y variadas dinámicas poblacionales. Para ilustrar este aspecto podemos recurrir a la contribución de los sociólogos que han utilizado las herramientas y los conceptos de la investigación demográfica, bajo el nombre de sociodemografía, para realizar estudios sobre matrimonio, divorcio y fertilidad (Richards, 1940; Mitchell, 1949; Barnes, 1949; Schapera, 1955; Colson, 1958; Ardener, 1962). Debido a que es la relación protagonizada por la demografía y la antropología la que nos interesa, profundizaremos más en este tema. 
El vínculo directo entre la antropología y la demografía no es muy temprano. Fue hasta las décadas de los setenta y ochenta del siglo pasado que los demógrafos se dieron cuenta de la importancia de los factores culturales en la dinámica de la población, percatándose de que el cambio en los fenómenos que matemáticamente recolectaban estaban profundamente ligados a factores culturales (religión, tradición, mitos, territorio y lenguaje, por mencionar algunos) (Leasure, 1963, p. 183). Para la década de los noventa, un enfoque se apuntalaba bajo el nombre de demografía antropológica, a partir de la discusión en congresos y la publicación del libro Anthropological demography (1997), en donde participaron de manera importante los antropólogos David I. Kertzer y Tom Fricke. Dicha perspectiva tiene la intención de establecer un puente teórico para enfrentar el reto interno de dos tradiciones epistemológicas y metodológicas distintas, que por el lado de la demografía suscribe los modelos causales universales y los estudios estandarizados; y por el de la antropología, siente una repulsión casi obsesiva e inexplicable por el manejo de números y ecuaciones estadísticas. A pesar de la coyuntura, ambas disciplinas se han unido en investigaciones interdisciplinarias, fortaleciéndose y reduciendo sus diferencias, actuando desde una plataforma en la que impera la relación entre la cultura, las diversas variables demográficas y la economía política.

De manera casi simultánea, surge la perspectiva de la antropología demográfica. Es importante mencionar que, si bien es cierto que los antropólogos desde un inicio han incluido en sus estudios información demográfica, ésta siempre ha sido de manera superficial, al grado de no concebirla como un enfoque distinto. No obstante, fue hasta finales de la década de los setenta, con mayor énfasis en las publicaciones de los años ochenta, que se marcó el inicio de un periodo en los estudios antropológicos con intereses demográficos, estableciéndose un nuevo paradigma denominado "antropología demográfica". Un ejemplo de su empleo en México fue el trabajo de Ada D’Aloja (1980, 1981, 1983 y 1997), quien realizó una investigación etnográfica con los temas centrales de migración, mortalidad y fecundidad de grupos otomíes del Valle del Mezquital, en Hidalgo, logrando explicar que los fenómenos que repercutían en la estructura de esta población indígena estaban ligados al entorno medioambiental y al comportamiento cultural. Asimismo, los resultados mostraron que el origen de algunas tradiciones se basaba en la creencia de la muerte de los recién nacidos y la necesidad materna de un nuevo embarazo de manera inmediata.

Cuestionamientos sobre prácticas culturales relacionadas a variables demográficas se encuentran de manera tácita en casi cualquier investigación antropológica. Explicaciones e implicaciones sobre el incremento o dismi- 
nución poblacional, guerras, enfermedades, migración, catástrofes naturales, distribución espacial, tamaño de asentamientos, son temas donde el enfoque de la antropología demográfica puede ser la clave.

A raíz de la discusión académica en un seminario llevado a cabo en la School of American Research en Nuevo México, en el año de 1973, se empezaron a formular las concepciones teóricas y metodológicas en las que estaría basado el naciente enfoque de la antropología demográfica -con objetivos distintos al planteado por los demógrafos décadas antes, llamado "demografía antropológica"-. Dichos resultados fueron publicados en 1978 con el título Demographic anthropology: Quantitative approaches, editado por el arqueólogo Ezra Zubrow.

Desde el punto de vista de este enfoque antropológico, el estudio de la dinámica poblacional no es simplemente un fenómeno cuantitativo o estadístico, ya que nos evidencia las características biológicas, económicas, políticas, sociales, religiosas y culturales que conforman la estructura de una población. Ésta no se puede explicar a través de formas reduccionistas, descrita matemáticamente con tasas, ecuaciones, gráficas, cuadros o tablas; el contexto espacio-temporal de las sociedades se enmarca en una dinámica donde interactúan diversos agentes. La antropología demográfica, durante las últimas cuatro décadas, ha conformado y desarrollado un conjunto teórico y metodológico distinto a otros: tiene la finalidad de estudiar los procesos y perfiles demográficos de las sociedades, puntualizando el aspecto cultural y el medioambiental, y teniendo a la estadística como uno de varios métodos de análisis.

Como hemos visto, el surgimiento y el desarrollo de la antropología demográfica son concebidos en términos del amplio campo antropológico y no como una propuesta nacida desde una sola de sus ramas, como lo plantean algunos antropólogos físicos en México (Hernández Espinoza y Márquez Morfín, 2003; Hernández Espinoza, 2006, 2010). Tanto la paleodemografía como la arqueología han desarrollado modelos con fuentes de información específicas y metodologías particulares con la finalidad de explicar la estructura de poblacionales del pasado, pero desde el enfoque amplio de la antropología demográfica. La paleodemografía, a partir del análisis de restos óseos, puede proveer importante información sobre las variables demográficas dinámicas como mortalidad, natalidad y migración, y su objetivo de estudio se centra en la caracterización de la historia individual y colectiva de los grupos humanos (Buikstra y Konigsberg, 1985, p. 316). En el caso particular de la arqueología, que utiliza un marco teórico-metodológico propio de la disciplina, proporciona información en torno a variables demográficas estáticas: distribución espacial, densidad poblacional, cálculos poblacionales 
y tasas de crecimiento, entre otras. Dicha perspectiva se ha denominado arqueología demográfica, sobre la cual profundizaremos a continuación.

\section{La propuesta de la arqueología demográfica}

La investigación arqueológica con temática demográfica tiene testimonios en la literatura desde hace aproximadamente cuatro décadas bajo el nombre de arqueología demográfica, y se inscribe en el debate intenso de una teoría antropológica con métodos arqueológicos y demográficos (Hassan, 1978, p. 50). Pretende conocer cualitativa y cuantitativamente los fenómenos demográficos de poblaciones extintas, valiéndose de la información recolectada a través de métodos arqueológicos - prospección, cartografía y excavación, entre otros-. Hassan, en su artículo "Demographic archaeology", propone un método para determinar el tamaño, la densidad y la tasa de crecimiento de las poblaciones del pasado, así como también destaca la importancia del uso de variables demográficas en las inferencias arqueológicas (Hassan, 1978, p. 49). Las preguntas que intenta responder esta perspectiva incluyen necesariamente la inferencia de supuestos en torno a la aparición, los cambios y la desaparición de las variables demográficas estáticas ya mencionadas; la relevancia de los factores económicos, políticos, culturales y religiosos en el sistema; las explicaciones adaptativas al entorno; las implicaciones de la subsistencia; los patrones espaciales; y las causas de la extinción de las poblaciones, por sólo enlistar algunas (Jochim, 1976; Wilmsen, 1973; Wobst, 1975; Keene, 1977). Esta perspectiva arqueológica concibe a las variables demográficas como las condicionantes en los sistemas culturales, aceptando que éstas no son determinantes, no juegan un papel independiente, ni se conciben como los motores principales de la evolución humana.

De hecho, uno de los planteamientos de la arqueología demográfica es la incorporación del método paleodemográfico como herramienta de inferencias demográficas en la medida de lo posible (Hassan, 1978, p. 51). Destacan entre las mayores contribuciones de ésta, la construcción e introducción de tablas de vida, modelos de población estable y modelos de población estacionaria (Ortega, 2004). Dentro de las numerosas e importantes investigaciones paleodemográficas desarrolladas a la par del nacimiento de esta propuesta arqueológica, se encuentran las efectuadas por Swedlund y Armelagos (1976), McKinley (1971), Angel (1969), Bocquet-Appel y Masset (1982), Hoppa (2002) y Mann (1975). Una publicación importante es la de Chamberlain (2006) sobre la revisión a la teoría y al método sobre reconstrucciones poblacionales con base en el dato arqueológico. Si bien es cierto 
que el libro examina fuentes etnográficas e históricas para estimar la demografía, la investigación se fundamenta en material osteológico y en su análisis biomolecular. En el caso particular de México, podemos mencionar los trabajos de Ortega (2004), Hernández Espinoza y Márquez Morfín (2003) y Hernández Espinoza (2006; 2010). No obstante, como ya hemos mencionado, el estudio paleodemográfico determina variables dinámicas de la población necesariamente a través del análisis de individuos. Por tanto, en sitios con una muestra osteológica deficiente o nula, la aplicación del método paleodemográfico se complica. Además, otro inconveniente es asumir que los restos óseos en un sitio representan a la población local, sin tomar en cuenta otras variables demográficas y culturales como la migración y las alianzas interregionales.

Las inferencias demográficas que se derivan de la investigación arqueológica tampoco responden a cuestionamientos sobre la demografía dinámica. Sin embargo, proporcionan otro tipo de deducciones correspondientes a la demografía estática, imposibles de revelar desde la paleodemografía: 1) tamaño, materiales constructivos y ubicación de las viviendas; 2) número, materiales constructivos y ubicaciones de las viviendas en el sitio; 3) número, tamaño, materiales constructivos y ubicación de los cuartos por vivienda, y 4) número de habitantes por cuarto, vivienda y sitio. Es decir, información trascendente que, junto a otras inferencias, posibilitan la reconstrucción de la compleja estructura poblacional (por ejemplo, nivel socioeconómico de los habitantes por vivienda; jerarquía social reflejada en los diferentes sectores del sitio, de acuerdo con su distribución espacial; ciclo de la unidad doméstica reflejada en el tamaño y materiales constructivos; presión de alta densidad poblacional sobre los recursos de subsistencia; papel de ciertos factores para el surgimiento de ciudades, urbes y estados; y componentes involucrados en la decadencia de las sociedades). Los trabajos arqueológicos interesados en conocer la demografía se han llevado a cabo en dos niveles: asentamiento y unidad doméstica.

\section{Nivel asentamiento}

Muy probablemente, el método para determinar el tamaño de la población por asentamiento más conocido y utilizado es el propuesto por Rauol Naroll (1962), quien, a partir de un estudio etnográfico del número de habitantes de 18 comunidades alrededor del mundo, propone una fórmula estadística para estimar el número poblacional de una sociedad extinta. La ecuación se fundamenta en el área total de superficie techada de las viviendas, llegando al 
resultado de que cada persona requiere un promedio de aproximadamente $10 \mathrm{~m}^{2}$ de espacio habitacional. Ejemplificando de manera hipotética y simple este método de proporción, podemos pensar que una vivienda con un área techada de $80 \mathrm{~m}^{2}$, probablemente fue habitada por ocho personas; por tanto, si en el asentamiento hay 20 viviendas del mismo tamaño, se puede derivar el tamaño de la población en aproximadamente 160 habitantes. Es importante mencionar que este método puede aplicarse a partir de la totalidad del área del asentamiento, o bien en alguna proporción del mismo; tal es el caso de conjuntos domésticos y unidades domésticas. No obstante, el fin será determinar el tamaño de la población total del sitio.

Desde nuestro punto de vista, se trata de un método demasiado sencillo para el complicado tema de la dinámica poblacional. Desde la perspectiva de quienes lo han aplicado, destaca: la insuficiencia en los datos para generar la fórmula (LeBlanc, 1971); el riesgo de subestimar o sobreestimar los resultados (Binford, Binford, Whallon y Hardin, 1970); y la consideración de que no toda el área techada se utilizó para vivienda (Soudsky, 1969). A lo anterior, hay que agregar la importancia de los factores medioambientales en la toma de decisiones para el establecimiento local. De acuerdo con el reporte de Millennium Ecosystem Assessment (MEA) de las Naciones Unidas, si examinamos el panorama poblacional en la actualidad, encontramos que hay mayor densidad poblacional en las regiones litorales con clima tropical y templado, mientras que las zonas con baja temperatura, obscuridad, aridez o calor húmedo y constante presentan un vacío demográfico y menor densidad (MEA, 2005). Los elementos topográficos, geomorfológicos, la altitud, la proximidad a los cuerpos de agua y la cercanía a los recursos de subsistencia juegan un papel importante en la organización espacial, el crecimiento y el número de la población (MEA, 2005). Por tanto, éstos no pueden ser resultado de una fórmula con coeficientes rígidos aplicables a las sociedades antiguas en todo el mundo.

Dentro de los estudios demográficos, con base en la propuesta de Narroll, se puede mencionar el desarrollado por P. Kardulias (1992, pp. 276-287) que, de acuerdo con la superficie de reposo en un asentamiento militar de Isthmania en Grecia, calcula $2.7-3.7 \mathrm{~m}^{2}$ por individuo y $1.8 \mathrm{~m}^{2}$ en condiciones de sobrepoblación; así como el de Pringle (1988), que estimó para África bizantina un espacio de 1.8-2.7 $\mathrm{m}^{2}$ por persona. Asimismo, es importante mencionar que existen reformulaciones a la constante de Naroll que exploran preguntas no elaboradas en el estudio previo, como qué es una vivienda, o cuál es la medida típica del área de piso para la vivienda de acuerdo con las características culturales; dicho replanteamiento resulta en un coeficiente de población mundial de $6 \mathrm{~m}^{2}$ por persona (Brown, 1987). Asimismo, Oliver y 
Gusi (1995, p. 220-221) plantearon una interpretación más restrictiva del método, tomando como base la superficie de cada vivienda en Puig de la Nao de Benicarló, dividiendo el área entre el tamaño total del asentamiento.

La alternativa para enmendar los huecos del método de proporción ha sido la aplicación de la etnoarqueología, es decir, el uso de datos etnográficos de lugares específicos con fines arqueológicos para la obtención de información relacionada con la cultura material de sociedades antiguas concretas. En el caso de los estudios mesoamericanos, ha permitido la obtención de coeficientes flexibles de acuerdo con los factores característicos del sitio de estudio (Redfiel y Villa Rojas, 1934; Cook y Heizer, 1968; Cook, 1972; Clarke, 1971; Sanders, 1962-3; Parsons, 1968; Haviland,1969; Puleston, 1973; Blanton, 1978; Laporte y Torres, 1988). Sin duda, uno de los trabajos más importantes ha sido el de Casselbery, basado en el estudio etnográfico de multifamiliares, donde se estima $6 \mathrm{~m}^{2}$ por individuo (Casselbary, 1974, pp. 117-122). Asimismo, se encuentra el de Kolb, quien realizó una revisión de los métodos para estimar el tamaño de la población a partir de indicadores particulares por sitio aplicables a asentamientos del área maya, teniendo como resultado $6.12 \mathrm{~m}^{2}$ por persona y el concepto de familia nuclear integrado por 5 o 6 miembros (Kolb, 1985, p. 585). Richard R. Wilk y Wendy Ashmore reunieron, en Household and community in the Mesoamerican past (1988), una serie de investigaciones arqueológicas sobre residencias y grupos domésticos ${ }^{1}$ en Mesoamérica, así como indicadores para su análisis. Por su parte, Zachary Nelson, en "De la cartografía al cálculo de población de Piedras Negras, Guatemala", conjuntó una lista de coeficientes poblacionales de varias sociedades modernas en el mundo, que van desde Chan Kom, Yucatán (5.6 personas por residencia) hasta Parma en Italia (4.16 personas por residencia) (Nelson, 2004, p. 7).

Aunque la etnoarqueología ha sido criticada por ser una "teoría de rango medio", debido a su incapacidad de probar hipótesis y por la tendencia entre los arqueólogos a pensarla como la fuente de perfectas analogías, este método ha sido de gran utilidad para la interpretación demográfica desde la arqueología, sirviendo para la formulación y el testeo de supuestos, así como para la propuesta de índices analíticos-imposibles de obtener mediante los tradicionales métodos arqueológicos-. El mismo Hassan (1978), en el ya mencionado artículo sobre arqueología demográfica, señala los problemas en torno a los cálculos poblacionales y hace hincapié en la necesidad de

1 Entendemos como "grupo doméstico" al grupo de personas que realizan actividades domésticas (preparar alimentos, comer, dormir, reproducirse, entre otras), de las cuales pueden quedar rastros materiales identificables por medio del estudio arqueológico. Dicho grupo puede compartir, o no, lazos de parentesco.

Estudios Demográficos y Urbanos, vol. 35, núm. 1 (103), enero-abril, 2020, pp. 117-152 
realizar investigaciones sistemáticas. El método de asignar un espacio por habitante conlleva como problemática básica obtener una cuantificación correcta de la superficie del asentamiento compuesto por áreas habitables y no habitables. Consideramos que los métodos de proporción, como el de Naroll, deben considerarse como parámetros de la estructura poblacional y, por tanto, una fase necesaria previa a los estudios de nivel unidad doméstica.

En los últimos años, con el apoyo de tecnologías de análisis geoestadístico y cuantitativo, se han empezado a desarrollar estudios demográficos que utilizan métodos para calcular la densidad poblacional de los sitios arqueológicos. Uno de los trabajos más representativos para Mesoamérica es el efectuado por Ortman, Cabaniss y Bettencourt (2013, p. 1), quienes analizaron los datos del asentamiento prehispánico en la Cuenca de México para mostrar que este sistema tuvo características espaciales análogas a la ciudad moderna. Por medio de una base de datos de 1400 sitios, ocupados durante dos milenios y caracterizados por un desarrollo político y socioeconómico distinto en cada periodo, se demostró que el tamaño de la población aumenta en una escala invariable con el rango $\mu=2 / 3-5 / 6$ de acuerdo con las expectativas de la teoría emergente y donde el límite inferior representa las formas simples de asentamiento y el superior a los sitios en los que la red de infraestructura determina la forma del área de asentamiento (Ortman, Cabaniss y Bettencourt, 2013, p. 2).

Con relación a los estudios demográficos a nivel asentamiento, y respecto al sitio sujeto al estudio de caso que aquí se presenta -el asentamiento olmeca de San Lorenzo, ubicado en la costa del Golfo-, podemos mencionar los trabajos en la sierra de los Tuxtlas (Sandey y Arnold, 1996; Santley, Arnold III y Barret, 1997), la Mixtequilla (Stark y Curet, 1994) y la Cuenca del Río Cotaxtla (Dannels, 1997). Métodos similares se utilizaron para los estudios del patrón de asentamiento en Oaxaca, donde se registró la cantidad de material arqueológico en superficie y la arquitectura (Balkansky et al., 2000; Blanton et al., 1979; Kowaleswki, Feinman, Finsten, Blanton y Nicholas, 1989). Asimismo, encontramos las estimaciones poblacionales basadas en la capacidad agrícola en el área maya, de Culbert y Rice (1990), o las investigaciones sobre comercio e intercambio interregional analizados por medio de la cerámica de Calakmul, por Domínguez y Folan (1999).

\section{Nivel de vivienda como unidad de conteo}

Los cálculos de la población de un asentamiento a través del cálculo de los habitantes por vivienda presentan una correlación más confiable que aquellos 
basados en el tamaño del asentamiento. Mediante el análisis demográfico de la vivienda podemos aproximarnos no sólo a las residencias y las funciones domésticas realizadas en ella, sino al grupo doméstico y a los componentes económicos, políticos y culturales de una parte de la sociedad. Este grupo representa la unidad social y económica más pequeña dentro del patrón de asentamiento. El análisis basado en esta unidad de conteo puede llegar a definir la estructura doméstica, el tamaño de ésta, la división del trabajo y la organización económica y política de la población, de acuerdo con cada sección del sitio. El método se basa en la identificación de unidades y/o conjuntos domésticos -que incluye el número y tipo de estructuras, así como los espacios abiertos; tal es el caso de posibles patios internos- por medio de la excavación. Es importante mencionar que, al igual que los métodos de cálculo poblacional a nivel asentamiento, conlleva limitantes. Determinar un coeficiente poblacional por estructura arquitectónica tiene como problemática definir cuál de éstas fue de uso habitacional y cuáles cumplieron otras funciones.

La aplicación de los métodos para calcular el tamaño de la población, ya sea a nivel asentamiento o nivel vivienda, dependerá de los supuestos de la investigación. En un punto ideal, ambas estimaciones deberán compararse con fines de obtención de tendencias. Orcutt (1974) comparó los cálculos del tamaño de la población a nivel asentamiento y a nivel vivienda en la región de Chevelon en Arizona, llegando a la conclusión de que el rango derivado de ambos métodos, aunque difiere -como es de esperarse-, es mínimo y representa información sistemática que posibilita la evaluación de diferentes trayectorias que inciden en la estructura y dinámica poblacional. Como se observará en el último apartado, donde se expone el caso de estudio en la capital olmeca de San Lorenzo, éste tuvo como objetivo calcular el tamaño de la población a lo largo del periodo Preclásico inferior (1800-1000 a. C.), con la finalidad de interpretar la estructura demográfica de una sociedad con características económicas, políticas, religiosas y culturales complejas. Para ello se aplicaron métodos distintos a nivel asentamiento y vivienda.

La visión y la propuesta de Hassan responden a la perspectiva general de los arqueólogos en el ámbito mundial durante las décadas de los setenta y los ochenta, quienes consideraban al crecimiento poblacional como factor clave en el surgimiento de cacicazgos y estados. Dicho enfoque no era ajeno al panorama arqueológico que se desarrollaba en México. En la arqueología mesoamericana, los estudios demográficos han tenido una íntima relación con los planteamientos que analizan la formación, el desarrollo y los cambios en los sistemas socioculturales. Como resultado de lo anterior, durante esa época se desarrollaron importantes investigaciones con la intención de ex- 
plicar los procesos de transformación que definen a las sociedades complejas como urbes y estados, donde se correlacionan fuertemente el aumento de la producción, el desarrollo de la agricultura intensiva, la alta densidad poblacional y el nivel de complejidad cultural (Sanders, Parsons y Santley, 1979).

Es importante mencionar que los estudios de patrón de asentamiento y el desarrollo de métodos de recorrido de superficie sistemáticos e intensivos, como el efectuado en la Cuenca de México por Sanders, Parsons y Santley (1979), aportaron datos sólidos que permitieron calcular la población antigua de México con relación al tamaño del sitio y a la cantidad de material arqueológico en superficie. Si bien es cierto que los estudios que derivan en cálculos poblacionales, como el antes mencionado, no se suscriben al enfoque de la arqueología demográfica apenas naciente en esa época, éstos fueron los principales promotores de una estrategia metodológica capaz de ser usada en los estudios sobre la estructura poblacional antigua a niveles regional, sitio e intrasitio.

En este sentido, conjuntando la aportación teórica propuesta por la arqueología demográfica y la aplicación de estrategias metodológicas para el estudio sistemático a nivel región, sitio y unidad doméstica -aunado, al aprovechamiento de nuevas tecnologías como los Sistemas de Información Geográfica (SIG) o imágenes Lidar, por mencionar algunas-, hoy en día podemos ser capaces de reconstruir la estructura demográfica (demográfica por su facultad de probar hipótesis y cuantificar variables) de poblaciones del pasado. El modelo de Hassan (1978) se sustenta en la propuesta de fuentes arqueológicas de información demográfica obtenidas a través de los distintos niveles de análisis en la investigación arqueológica. Aunque consideramos que dicho método continúa siendo viable, para fines de este artículo le hemos añadido la práctica interdisciplinaria y el uso de nuevas tecnologías, lo que resultará en la aplicación sistemática del enfoque teóricometodológico de la arqueología demográfica.

No podemos dejar de mencionar que una de las mayores críticas al enfoque mencionado ha sido la preocupante y frecuente referencia a la propuesta de cálculos poblacionales como un fin; es decir, la presentación de un número de habitantes como el resultado del trabajo de investigación, sin mostrar las diversas causas y numerosas consecuencias que conlleva ese conglomerado de personas. Esta acusación se vuelve injusta al revisar las bases en las que está cimentado el enfoque. En este sentido, la exposición de un cálculo poblacional, así sea el obtenido por el método más riguroso, no implica la aplicación del modelo de la arqueología demográfica si éste no viene aparejado necesariamente a una serie de explicaciones y consecuencias sociales. Es necesario definir, desde el inicio, los objetivos, el diseño y las 
estrategias de exploración para producir información y datos apropiados para el análisis demográfico desde la arqueología. A continuación, expondremos un estudio de caso que muestra el potencial del enfoque.

\section{Estudio de caso: arqueología demográfica en la primera capital olmeca de San Lorenzo, Veracruz}

La capital olmeca de San Lorenzo se ubica en la costa del Golfo de México, en el sur del estado de Veracruz -uno de los puntos de selva húmeda tropical y pantanosa del país- (Mapa 1). Tiene su origen alrededor de 1800 a. C., y tuvo un desarrollo ininterrumpido durante ocho siglos, llegando a abarcar 776 hectáreas. Aunque los primeros reportes sobre la cultura olmeca se llevaron a cabo desde finales del siglo XIX, fue hasta 1939 que se realizaron los hallazgos en el sitio por el arqueólogo Matthew W. Stirling, donde destacan cabezas colosales y tronos. El centro de la capital de San Lorenzo se conforma por una gran meseta, una construcción artificial que representa, hasta el momento, la primera obra hecha de tierra apisonada en Mesoamérica. Alrededor de 1200 a. C., durante la fase de auge del asentamiento, los habitantes aplicaron siete millones de metros cúbicos de relleno de tierra para conformar un sitio en el cual asentarse en medio de una planicie aluvial rodeada de agua proveniente de las lluvias típicas y el desborde de afluentes del Coatzacoalcos (Cyphers et al., 2014). San Lorenzo se caracteriza por su compleja estratigrafía y arquitectura manufacturada de tierra, lo que ha implicado la necesaria aplicación de métodos y modernas tecnologías que ayudan a la realización de nuevas interpretaciones sobre este importante sitio del periodo Preclásico inferior (Cyphers et al., 2014).

Con el fin de examinar la antigua dinámica poblacional de San Lorenzo, con base en el tamaño y la configuración del asentamiento y de las casas olmecas, se llevó a cabo un recorrido de superficie con la meta de aproximar la extensión horizontal del asentamiento y de su población (Symonds, Cyprers y Lunagómez, 2002); y posteriormente, efectuar un programa de excavaciones y pruebas con barrenos para la identificación de estructuras arquitectónicas y derivación del tamaño y densidad de las viviendas a nivel intrasitio (Arieta y Cyphers, 2017a; Arieta, 2013). A continuación, haremos una breve descripción de los estudios antes mencionados, correlacionándolos con la propuesta metodológica de la arqueología demográfica (Hassan, 1978), mostrando sus derivaciones y exponiendo cómo la comparación de resultados proporciona inferencias demográficas confiables en torno a sociedades del pasado, en este caso la olmeca. 


\section{Mapa 1}

Ubicación geográfica del sitio arqueológico de San Lorenzo, Veracruz

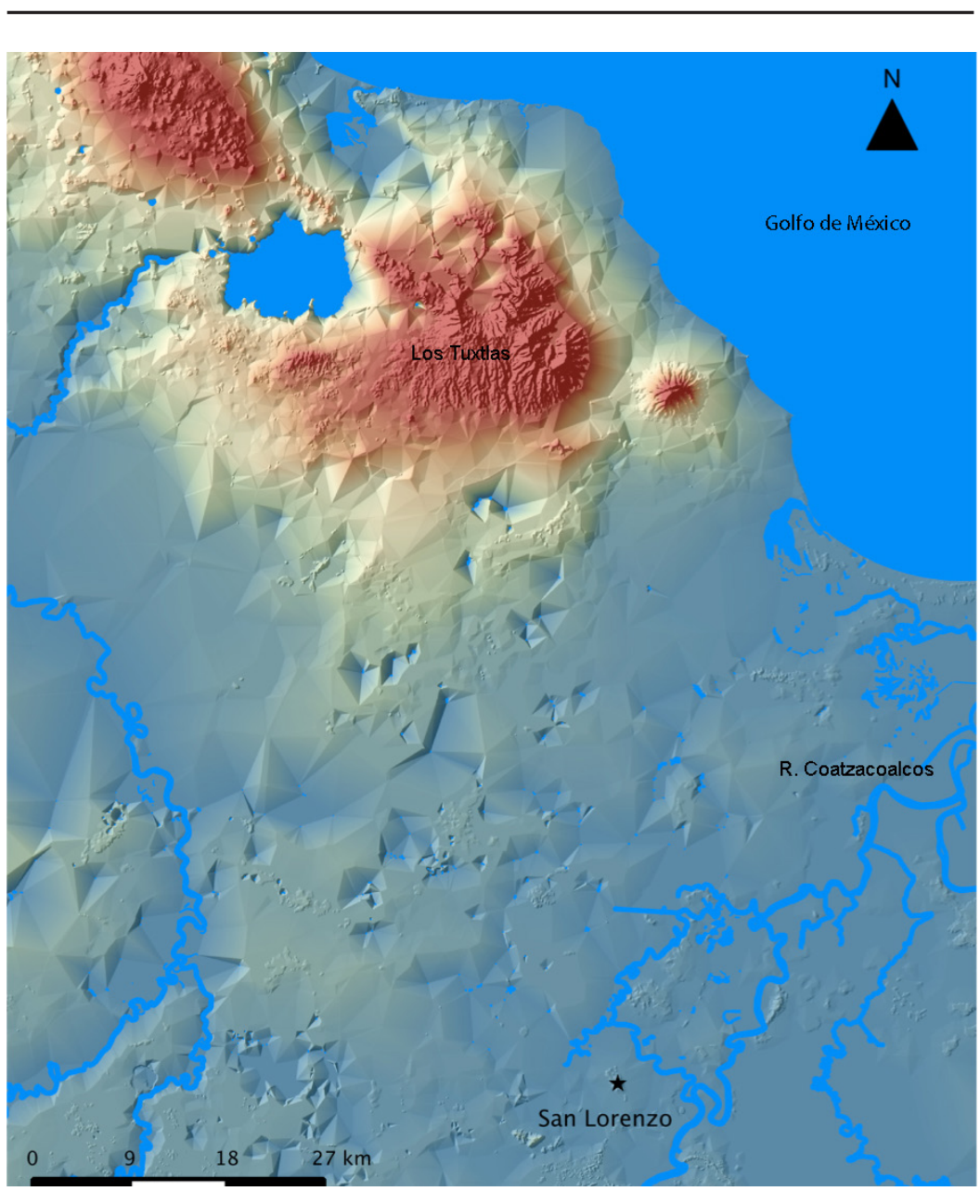

Fuente: Plano de Virginia Arieta Baizabal. 
El primer nivel del modelo de la arqueología demográfica de Hassan (1978) consiste en el análisis regional a través de los estudios del patrón de asentamiento, tomando en cuenta el aspecto medioambiental, geomorfológico y morfológico de los sitios (Esquema 1). Los recorridos regionales, de carácter sistemático e intensivo alrededor de San Lorenzo, retomaron la idea de que los estudios de densidad poblacional están estrechamente relacionados con el tema del patrón de asentamiento por la intrínseca relación entre el entorno, la organización social y la arquitectura. El estudio del ambiente fisiográfico realizado por el Proyecto Arqueológico San Lorenzo Tenochtitlán (PASLT) se ha llevado a cabo mediante la aplicación de diversas técnicas y metodologías de estudio. Uno de los primeros pasos fue la reconstrucción de procesos y formas del antiguo paisaje efectuado por Ortiz y Cyphers (1997). Los autores definieron las unidades geomorfológicas en la región y la secuencia de su desarrollo identificando antiguos cauces fluviales presentes durante el Preclásico, ahora extintos (Ortiz y Cyphers, 1997, p. 40). Se demostró que San Lorenzo se ubicaba en un lugar estratégico, a salvo de inundaciones y rodeado de vías para la comunicación y el comercio. Lo anterior, se corroboró décadas más tarde, con la identificación de un antiguo puerto fluvial en asociación a un taller de obsidiana localizados en la parte sur del asentamiento (Cyphers y Hirth, 2016).

El análisis de los restos de plantas y animales recuperados en las excavaciones también ha ocupado un lugar importante dentro de las investigaciones en San Lorenzo. Éstos permitieron reconocer los patrones de explotación del entorno. Particularmente, la aplicación de la arqueometría ha posibilitado la identificación de polen y fitolitos, mostrando las relaciones de los pobladores del Preclásico inferior con el ambiente para la subsistencia (Zurita, 1997; Cyphers, Zurita y Lane, 2013). Específicamente, la investigación de Cyphers, Zurita y Lane tuvo como resultado la identificación de áreas dentro de los humedales ubicados al norte del asentamiento, utilizadas para la obtención de alimentos acuáticos como complemento a la cosecha de tubérculos (Cyphers, Zurita y Lane, 2013, pp. 13-14). Dicho estudio incluye importantes inferencias demográficas para las etapas de auge. La presión poblacional en la isla de San Lorenzo debió crear mayor necesidad de alimentos y de abastecimiento para la etapa de crisis, lo que muy probablemente llevó a una competencia y, consecuentemente, a la decadencia de la primera capital (Cyphers, Zurita y Lane, 2013, p. 14).

Las primeras reconstrucciones geomorfológicas y medioambientales de San Lorenzo prepararon el terreno para las observaciones del programa de recorrido de superficie, correspondiente a los primeros niveles en el modelo de Hassan (Esquema 1). Dicho estudio aportó cálculos poblacionales 


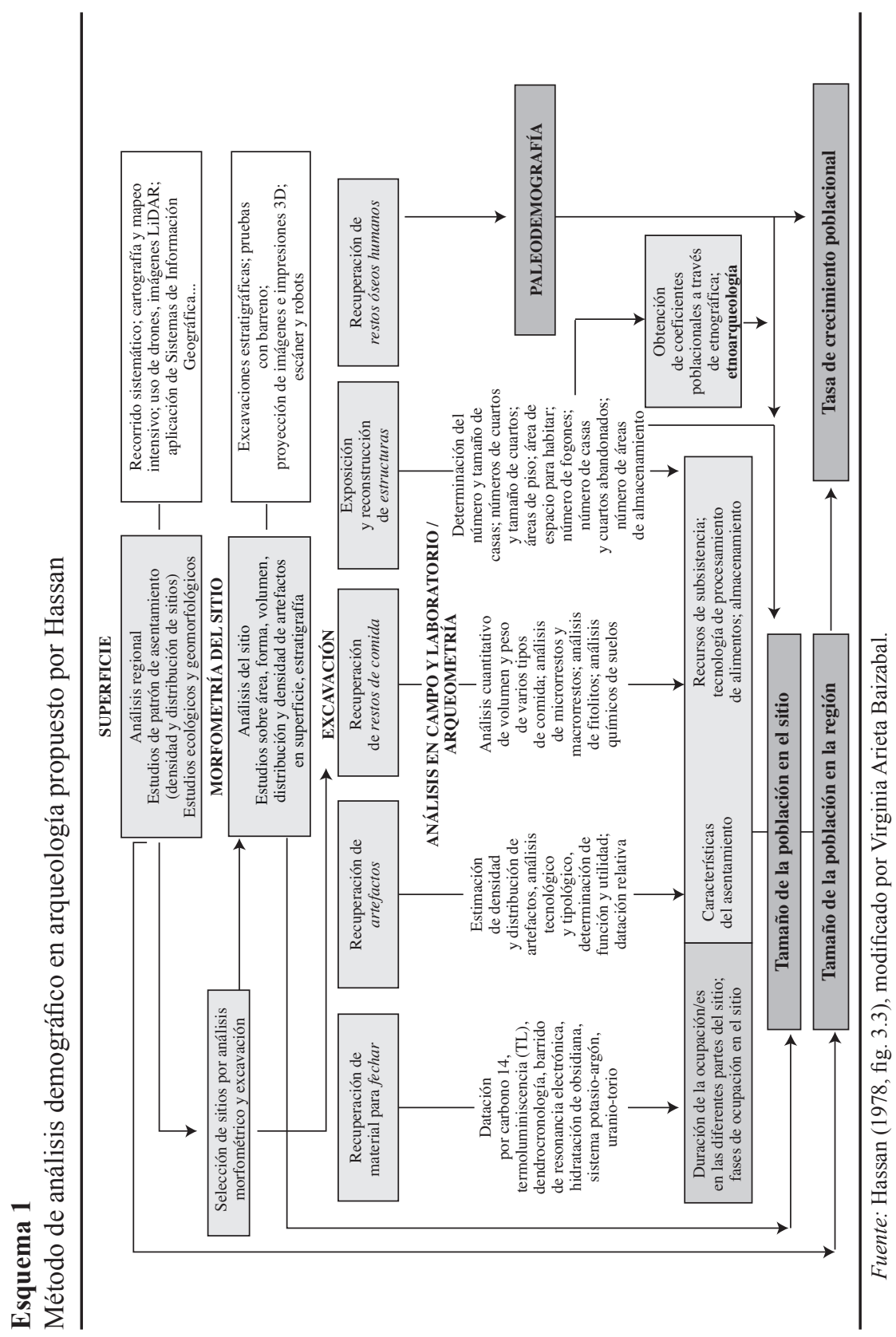


confiables y el establecimiento de ocho niveles de tipo de sitio, definidos con base en la distribución de material arqueológico, el tamaño y la distribución de la arquitectura (Symonds, Cyphers y Lunagómez, 2002, p. 39). Según el estudio del patrón de asentamiento y los cálculos demográficos, San Lorenzo tuvo a nivel regional la siguiente dinámica poblacional (Symonds, Cyphers y Lunagómez, 2002): durante las fases tempranas (c. 18001400 a. C) se registraron 105 sitios con una población entre 426 y 1017 habitantes (mediana: 722); de ellos, 81 se encuentran cerca de San Lorenzo y 24 tienen un patrón disperso dentro del área de estudio. El sitio mayor, San Lorenzo, se encuentra en un terreno elevado, rodeado por antiguas vías fluviales y tiene un tamaño aproximado de 20 ha (Symonds, Cyphers y Lunagómez, 2002, p. 58). Para la etapa de auge, había una fuerte tendencia ocupacional en el hinterland interior, con un número de $6952-15022$ habitantes (mediana: 10 987). Según el estudio del patrón de asentamiento, San Lorenzo tenía una distribución habitacional en la cual el espaciamiento entre las unidades domésticas variaba de 25 a $50 \mathrm{~m}$ en la parte central del sitio, 100 a $150 \mathrm{~m}$ en las terrazas, y $200 \mathrm{~m}$ en la periferia (Symonds, Cyphers y Lunagómez, 2002, pp. 48-49). Una reconsideración posterior en el tamaño del sitio por Cyphers et al. (2007-2008) derivó en una población mínima de 4900 y una máxima de 10500 habitantes. Dichos cálculos se verán fortalecidos con los resultados del programa de excavaciones y pruebas con barreno que a continuación se describen.

La recuperación de artefactos, la exposición y la reconstrucción de estructuras - determinación del número y tamaño de casas, cuartos, áreas de pisos, fogones y áreas de almacenamiento- a través de excavación, representan el siguiente nivel dentro del modelo de la arqueología demográfica (Esquema 1). En San Lorenzo, Coe y Diehl fueron los primeros en proporcionar cálculos poblacionales a nivel intrasitio, a partir del mapa realizado por Krotser, donde se detectaron 200 montículos bajos (Coe y Diehl, 1980, vol. 1, pp. 29-30). Éstos, al ser considerados de uso habitacional, sirvieron como base para la primera estimación poblacional del sitio olmeca a nivel vivienda, resultando un número de 1000 habitantes para San Lorenzo (Coe y Diehl, 1980, vol. 1, pp. 29-30). Posteriormente, al comprobar que los montículos bajos no eran construcciones olmecas, sino que correspondían al periodo Clásico tardío-terminal, se rechazó dicho cálculo (Cyphers, 1997; Hernández, 2000).

La demografía olmeca a nivel vivienda continuaba siendo una incógnita. Como ya hemos mencionado, las características de la arquitectura preclásica de San Lorenzo desafían a la arqueología debido a la complejidad que conlleva una estratificación profunda (de hasta 20 metros) y un sistema 
constructivo basado en edificios hechos completamente de tierra. A raíz de lo anterior, se llevó a cabo una investigación que involucró tres rubros: 1) el programa de excavaciones y pruebas de barreno, tratándose del método principal de donde se obtuvo toda la información estratigráfica necesaria para la identificación de las viviendas olmecas; 2) los SIG, una de las tecnologías de la información más poderosas que se han desarrollado para el análisis de cuestiones relacionadas con el espacio geográfico; y 3) la realización de un estudio etnográfico en la comunidad de Tenochtitlán -cercana al sitio arqueológico- para la obtención de coeficientes poblacionales de acuerdo con las características medioambientales y de subsistencia en la región (Arieta y Cyphers, 2017b).

Respecto al primer y segundo puntos, se llevó a cabo un programa sistemático de pruebas con barreno (2 602 pruebas) durante los años 2005 a 2007 (Mapa 2). Esta estrategia metodológica proporcionó cuantiosa información, constituida por 25820 estratos, que requirió el uso de SIG para el análisis de datos y la presentación de resultados. Cabe destacar que el método y la aplicación del programa de pruebas con barreno en San Lorenzo, así como las observaciones preliminares, se encuentran en la obra Atlas digital de la zona arqueológica de San Lorenzo, Veracruz (Cyphers et al., 2014). No obstante, para los fines de este artículo, es importante hacer mención del procedimiento que se utilizó para la identificación de viviendas olmecas y de los métodos de estimación demográfica a partir del registro arqueológico, pues son parte de nuestros argumentos centrales.

Las propiedades físicas de los suelos conformaron el principio de sistematización, logrando identificar las superficies ocupacionales, según lo observado en las excavaciones previas, de las cuales se obtuvo una muestra considerable de pisos presentes en el sitio. Según las excavaciones, los pisos corresponden al interior de las estructuras, ya que hay muros que los delimitan, son horizontales, de grosor homogéneo y están elaborados de materiales particulares; tal es el caso de la hematita, bentonita y arenas (Cyphers, 2012). Cabe destacar que dichos materiales constructivos aportan información socioeconómica donde está involucrado fuertemente el factor demográfico, como más adelante describiremos.

Esta clasificación se correlacionó estratigráficamente con la identificación de etapas constructivas con temporalidades distintas (Cyphers et al., 2007-2008). Por tanto, cada estrato distribuido en las 2602 pruebas tiene una correspondencia temporal dentro de las fases constructivas: Arenas (c. 1800-1400 a. C.), Terrazas (c. 1400-1200 a. C.) y BAV (c. 1200-1000 a. C.); está última caracterizada por un suelo arcillo-arenoso amarillento (Arieta y Cyphers, 2017a, p. 64).

Estudios Demográficos y Urbanos, vol. 35, núm. 1 (103), enero-abril, 2020, pp. 117-152 


\section{Mapa 2}

Conjuntos domésticos y viviendas aisladas, resultado del análisis de los pisos mediante SIG

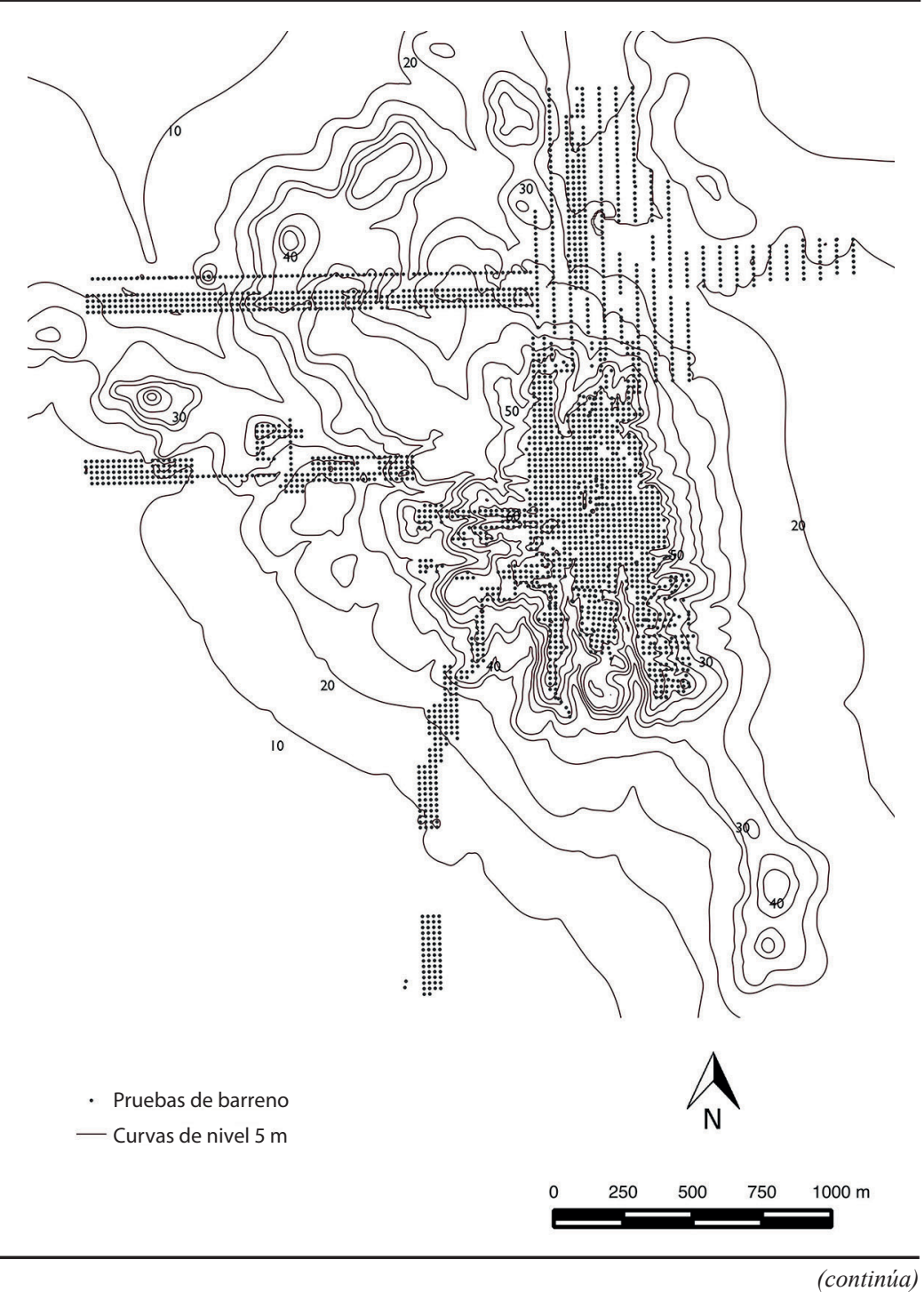




\section{Mapa 2}

(concluye)

Ubicación de las 2602 pruebas con barreno

efectuadas en el sitio arqueológico

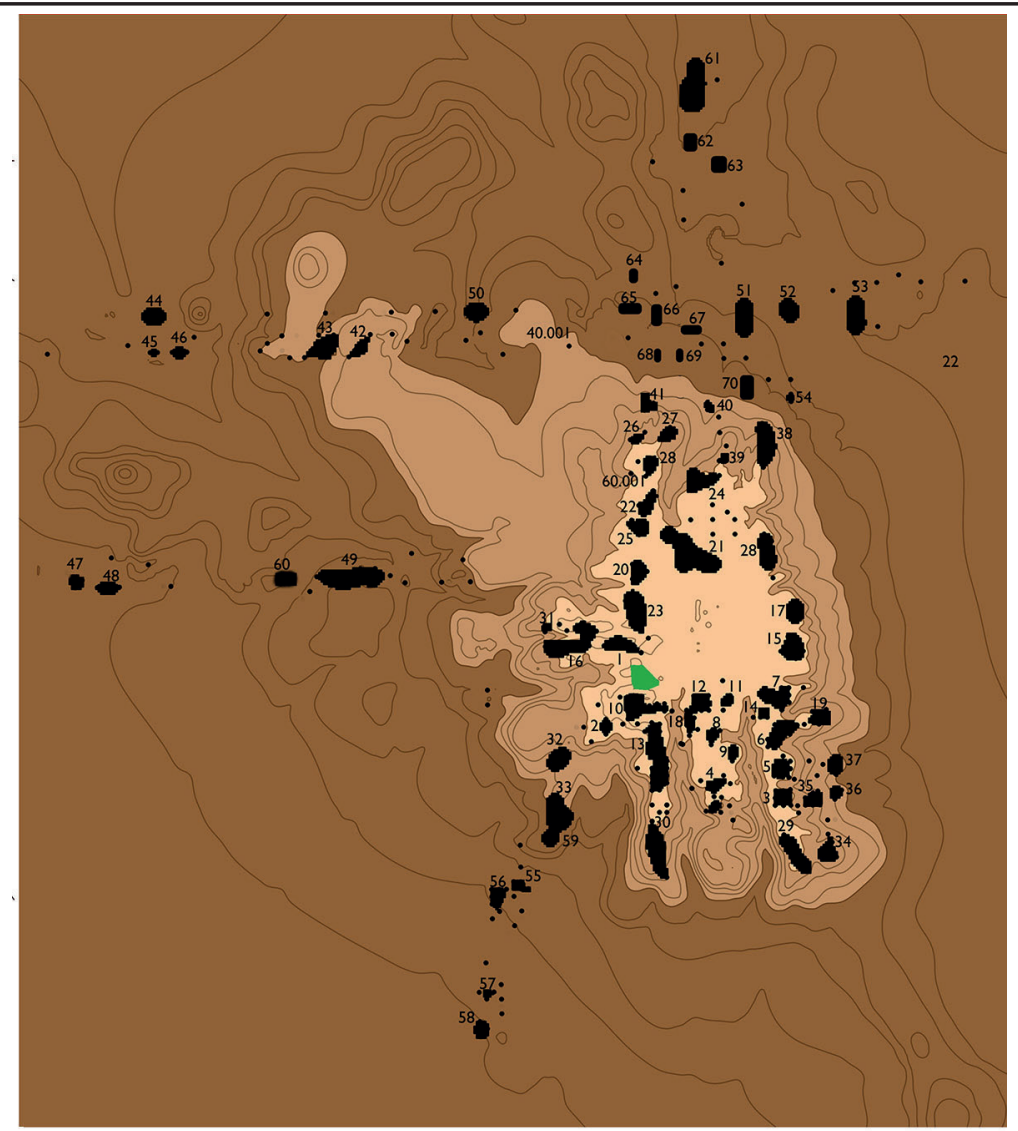

Concentraciones de dos o más pruebas de barreno con pisos

$\square$ Concentración Grupo $\mathrm{E}$

- Pruebas de barreno con pisos independientes

- Curvas de nivel $5 \mathrm{~m}$

Periferia (40-22 msnm)

Terrazas (60-40 $001 \mathrm{msnm})$

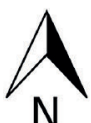

Cima (65-60 $001 \mathrm{msnm})$

Fuente: Tomada de Arieta y Cyphers (2017a, figura 3.3), modificado por Virginia Arieta Baizabal.

Estudios Demográficos y Urbanos, vol. 35, núm. 1 (103), enero-abril, 2020, pp. 117-152 http://dx.doi.org/10.24201/edu.v35i1.1851 
De acuerdo con el modelo de la arqueología demográfica de Hassan (1981), los pisos obtenidos mediante el programa de prueba con barreno en San Lorenzo son evidencia clave que permite determinar el número y el tamaño de los edificios y, por tanto, una aproximación a la estructura poblacional vista a través del registro arqueológico. En las 2602 pruebas se identificaron 1651 pisos de estructuras, mismos que se analizaron tomando en cuenta el contenido de tres variables básicas: 1) espacio (UTM de cada prueba con barreno en frentes de excavación, parcelas, áreas del sitio), 2) tema (tipo de suelo, espesor, profundidad), y 3) tiempo (fase ocupacional, etapa constructiva).

Para el estudio de la densidad poblacional por medio de los pisos fue fundamental la realización de mapas con variables cuantitativas a través del análisis geoespacial. La herramienta principal fueron los mapas de calor, también llamados mapas de concentración, los cuales representan gráficamente información mediante la gradación de color. De esta forma, los mapas de calor son representaciones gráficas de datos cuyos valores individuales están contenidos en una matriz y se representan con colores, intensidades y tamaños; la gradación intensa expresa diversos intervalos de un fenómeno en unidades espaciales (Arieta y Cyphers 2017a, p. 64). Dichos mapas permiten identificar fácilmente excepciones e información relevante que no se ve a simple vista con otro tipo de análisis. Es decir, el análisis de las pruebas con barreno por medio de mapas de calor nos permite identificar concentraciones en donde hay presencia de pisos y concentraciones de éstos por tipo de suelo. Estas agrupaciones estarán representadas de acuerdo con diversos intervalos o perímetros según nuestros objetivos.

Se realizó una serie de ejercicios con mapas de calor para cada etapa constructiva. El análisis cuantitativo y geoestadístico sobre la densidad de estructuras habitacionales se llevó a cabo tomando en cuenta las áreas recurrentes con sobreposición de pisos, como es el caso del sur del asentamiento, y el material con el que está elaborado el piso (hematita, bentonita, grava, arena, entre otros). Posteriormente, se obtuvieron medidas de área de cada una de las concentraciones de pisos con ayuda de la herramienta de polinización del QGIS de OSGeo (Arieta y Cyphers 2017a, tabla 1). La determinación del tamaño del área de concentraciones de pisos en San Lorenzo, aspecto señalado por Hassan (1978) en su modelo demográfico, fue el punto de partida para la inferencia del cálculo de densidad de estructuras arquitectónicas y, con base en coeficientes obtenidos en un estudio etnográfico, para la estimación poblacional del sitio.

Antes de pasar a ellas, describiremos brevemente la implementación del método etnoarqueológico en San Lorenzo, que si bien es cierto que ha 
sido utilizado desde tiempo atrás por los arqueólogos en busca de fuentes precisas de analogía - punto donde recae una de sus mayores críticas-, en el caso de San Lorenzo ha mostrado ser una herramienta viable, en conjunción con otras, para la derivación de datos comparables y, por tanto, un método viable para la reconstrucción como parte del enfoque de la arqueología demográfica.

Como se ha señalado, la aplicación de coeficientes poblacionales, obtenidos por medio del estudio etnográfico de poblacionales actuales, a los datos del patrón de asentamiento prehispánico es un método ampliamente ocupado por los arqueólogos interesados en los estudios de densidad poblacional y distribución espacial. A pesar de que este tipo de investigaciones conlleva grandes obstáculos, como la posible ausencia de información escrita, la formulación de hipótesis que ayuden a responder cómo una población ocupa, utiliza y aprovecha el espacio físico donde habita y se desarrolla se convierte en una fuente de información alterna que proporciona coeficientes factibles. La mayoría de los coeficientes utilizados para realizar estimaciones poblacionales de sociedades antiguas ha sido obtenida por medio de la etnografía y de los censos históricos y modernos. Los aspectos demográficos prehispánicos con base en estudios etnográficos han tenido gran popularidad en el área maya debido a que varios sitios o comunidades cercanas a los asentamientos arqueológicos presentan una historia ocupacional continua desde la época prehispánica (Redfield y Villa Rojas, 1934; Sanders, 19621963; Puleston, 1973; Zachary, 2004; Haviland, 1969; Parsons, 1968).

Para San Lorenzo no se había aplicado un enfoque etnográfico que derivara en datos comparativos basados en la densidad poblacional de una sociedad de nuestros días asentada en el asentamiento prehispánico. Por ello, se formuló un estudio enfocado en el poblado moderno de Tenochtitlán, Veracruz, el cual ocupa el mismo terreno donde se asentaron los olmecas durante el Preclásico inferior (Arieta y Cyphers, 2017b). La ubicación del poblado a sólo dos kilómetros del asentamiento arqueológico, ambos en la isla de San Lorenzo, resalta su extraordinaria relevancia en la problemática de la demografía olmeca, a pesar de la gran diferencia temporal y los cambios en las condiciones medioambientales. Dicho estudio tuvo como principal objetivo comprender cómo la población moderna utiliza el espacio cuando las características medioambientales y de subsistencia pudieron ser compartidas con los antiguos pobladores del asentamiento prehispánico (Arieta y Cyphers, 2017b, p. 9). La investigación en Tenochtitlán se llevó a cabo a nivel comunidad, nivel solar y nivel vivienda, lo que permitió identificar una densidad poblacional nucleada en el centro y dispersa en la periferia (Arieta y Cyphers, 2017b, pp. 12-13). Lo anterior tiene gran relevancia ya que ambas 
sociedades -la antigua y la actual-desarrollaron modelos similares a la hora de tomar decisiones de distribución espacial, como observaremos más adelante. Asimismo, se obtuvieron coeficientes específicos comparativos a la sociedad olmeca. Las autoras hacen hincapié en el hecho de que el tipo de grupo doméstico presente en la época prehispánica no es igual que el tipo de grupo doméstico de las sociedades actuales. No obstante, el coeficiente poblacional derivado del estudio etnográfico en Tenochtitlán puede ser lo suficientemente factible para ser aplicado en la estimación poblacional para San Lorenzo por compartir el mismo espacio (Arieta y Cyphers, 2017b, p. 20). Asimismo, se evita recurrir a datos de estudios hechos en el área maya $u$ otra región que posiblemente nada tengan que ver con la sociedad olmeca. El coeficiente poblacional de la comunidad de Tenochtitlán es de 9 a 16 habitantes por solar, el cual se compone de dos o más viviendas ocupadas por familias nucleares emparentadas (Arieta y Cyphers, 2017b, p. 20). Este dato forma el elemento complementario para estimar el número de habitantes con relación al área que ocupan los conjuntos y unidades domésticas observados en el análisis geoestadístico de las pruebas con barreno arriba mencionado.

De esta manera, el estudio del programa de pruebas con barreno, en conjunción con la investigación etnoarqueológica, permitieron llegar al último nivel propuesto en el modelo de la arqueología demográfica (Esquema 1): reconstruir el número y el tamaño de las estructuras arquitectónicas ubicadas en los diversos sectores del sitio y, a partir de éstos, calcular el total de los habitantes en el asentamiento con el fin de llegar a inferir tendencias en la dinámica poblacional, en este caso sobre la primera capital en Mesoamérica desde 1800 a 1200 a. C. (Arieta y Cyphers, 2017a; 2017b; s.f.). Como resultado se obtuvieron tendencias sobre el establecimiento local e interpretaciones en torno a los aspectos demográficos y socioeconómicos de esta sociedad olmeca, como los que a continuación se anotan brevemente.

Para las fases más tempranas (1800 a. C.), los pobladores de San Lorenzo eligieron para vivir la parte más alta y central de la meseta. Alrededor de 1200 personas habitaban en la que era una aldea grande de aproximadamente 56 ha, de acuerdo al análisis geoestadístico de los pisos y los materiales arqueológicos presentes en las pruebas con barreno. Lo que se ha podido observar, mediante el análisis de pisos, es que el asentamiento estaba conformado por 27 conjuntos habitacionales -tres o cuatro casas alrededor de un patio, según lo evidenciado en las excavaciones extensivas y similar a lo observado en el estudio etnográfico de Tenochtitlán- y 57 viviendas independientes con un tamaño promedio de $200 \mathrm{~m}^{2}$. Se identificaron alrededor de once tipos de pisos, siendo los manufacturados en grava y arena, bentonita y rojos de hematita los de mayor uso (Arieta y Cyphers, s.f.). 
De acuerdo con el ya mencionado estudio de Cyphers et al. (2007-2008) sobre la identificación de etapas constructivas de la meseta de San Lorenzo, en la etapa siguiente (1400-1200 a. C.) hubo una intensa actividad constructiva cuando los habitantes modificaron el terreno mediante la aplicación de seis millones de metros cúbicos de relleno para la construcción de terrazas habitacionales. Lo anterior influyó en la distribución y la organización de aproximadamente 3500 habitantes asentados en un área de 195 ha (Arieta y Cyphers, s.f.). Se identificaron 50 conjuntos domésticos ubicados únicamente en el terreno alto, lo que muestra una estrecha relación entre el estatus y la altura del terreno. Además, ocuparon 200 viviendas independientes localizadas en las elevaciones intermedias y los alrededores, lo que evidencia un ligero desplazamiento de la población del centro hacia las terrazas habitacionales. Se mantuvieron como populares los pisos hechos en bentonita, grava y arena, y rojos de hematita (Arieta y Cyphers, s.f.).

La etapa de auge (1200-1000 a. C.) se refleja con el incremento en el número de pisos (1 051) y por ende, con el aumento de la población, distribuida en la cima, las terrazas y la periferia. El tamaño del sitio y de la población aumentaron exponencialmente, llegando a abarcar 775 ha donde habitaban alrededor de 12000 personas (Arieta y Cyphers, 2017a, p. 70). Más de 300 conjuntos habitacionales y 481 viviendas aisladas se distribuían por todo el asentamiento (Mapa 2). En la cima y en las terrazas habitacionales únicamente se ubicaba el $26 \%$ de las viviendas independientes; la periferia estaba ocupada por más del $74 \%$. Sucedía lo mismo con los conjuntos domésticos; no obstante, es importante hacer notar que su número era inversamente proporcional a su tamaño, según el sector. Es decir, el número de conjuntos era mayor en la periferia, pero en la cima se localizaban los de mayor tamaño, que alcanzaban los $6500 \mathrm{~m}^{2}$, y los construidos con materiales de prestigio (Arieta y Cyphers 2017a, p. 68). Doce tipos de piso fueron identificados para esta etapa, destacándose los de bentonita, grava y arena, rojos de hematita, café o gris y los manufacturados en arenas de diversos colores (Arieta y Cyphers, s.f.).

Hasta este punto, en San Lorenzo han sido realizados diversos estudios con objetivos demográficos bajo diversos métodos y cubriendo cada uno de los niveles propuestos en el modelo de la arqueología demográfica. Éstos han arrojado diferentes estimaciones poblacionales que concuerdan en algunos de sus rangos: la propuesta por Symonds, Cyphers y Lunagómez (2002, pp. 47-50), a partir del reconocimiento de superficies y con base en un cálculo de tamaño de sitio de 500 ha, derivó en un rango poblacional de entre 3500 y 7500 habitantes para la isla. Una reconsideración posterior en el tamaño del sitio (700 ha) por Cyphers et al. (2007-2008) arrojó una población 
mínima de 4900 y una máxima de 10500 habitantes. Posteriormente, fundamentado en la aplicación del coeficiente poblacional, obtenido del estudio etnográfico de la comunidad de Tenochtitlán ( 9 a 16 habitantes por solar, el cual estaba constituido por dos o más viviendas) al área de las concentraciones de pisos identificados en las pruebas con barreno, se obtuvo una estimación de entre 5169 y 9161 habitantes (mediana: 7164 pobladores) (Arieta y Cyphers, 2017b). Finalmente, el análisis geoestadístico de las pruebas con barreno realizado mediante SIG de los pisos de las viviendas, arrojó una estimación para la etapa de auge de 10413 habitantes (Arieta, 2013). Con base en lo anterior, se puede considerar factible que la población de San Lorenzo en 1200 a. C. podría ubicarse entre los 9000 y 10000 habitantes.

El tamaño de la población en la región y el sitio, así como la tendencia de crecimiento, representan el último nivel en el modelo propuesto por Hassan (1978) para la reconstrucción demográfica desde la arqueología. No obstante, queremos destacar que este último eslabón no significa la reconstrucción en la dinámica poblacional. Es decir, el cálculo poblacional sólo es la base que, junto a otros factores, ayuda a exponer las explicaciones e implicaciones sobre el crecimiento y la disminución de la población, entre otros factores socioeconómicos.

La determinación del tamaño y la densidad poblacional en San Lorenzo, llevadas a cabo a la par de otras investigaciones que versan sobre la reconstrucción del medio ambiente y la subsistencia, han permitido conocer más sobre la dinámica poblacional olmeca en el sitio. Juntas fortalecen el supuesto de que el aumento poblacional durante las fases más tempranas del sitio (1800 a. C.) disminuyó la cantidad de tierras potencialmente cultivables, lo que propició la aparición e intensificación de otros medios de sobrevivencia, logrando así que el asentamiento llegara a su mayor esplendor en las etapas siguientes, particularmente en la fase arqueológica denominada San Lorenzo B (c. 1200-1000 a. C.), y se convirtiera en la primera gran capital de Mesoamérica. Posteriormente, el aumento de población y la disminución de recursos -observados en el análisis de fitolitos y polen (Cyphers, Zurita y Lane, 2013)provocaron presión demográfica, empobrecimiento, disgustos internos, enfermedades, migración, entre otras tendencias que derivaron en la decadencia del sitio (1000- 900 a. C.).

El presente estudio de caso sirve para mostrar una serie de análisis correlacionados con la propuesta metodológica de la arqueología demográfica (Hassan, 1978), que expone sus derivaciones y exhibe cómo la comparación de resultados proporciona inferencias demográficas confiables en torno a sociedades del pasado. Reiteramos las limitaciones sobre las estimaciones poblacionales de la arqueología, pero se ofrecen coeficientes preliminares y 
planteamientos sobre la importancia de realizar investigaciones interdisciplinarias con objetivos demográficos.

\section{Observaciones finales}

El surgimiento del enfoque de la antropología demográfica tiene un carácter intradisciplinario, interdisciplinario y transdisciplinario, que constituye un marco teórico-metodológico complementario y que tiene como fin explicar las variadas perspectivas de un mismo fenómeno; tal es el caso de la dinámica poblacional. Los objetivos y objetos de estudio de la antropología física y la arqueología abren un espacio de convergencia y acercamiento a una realidad de grupos humanos extintos, a través de la paleodemografía y la arqueología demográfica. Ambas disciplinas antropológicas pueden tener distintas perspectivas de un mismo problema de estudio, tan distintas como las que pueden tener con otras disciplinas científicas; y está bien, porque de esta manera se complementan.

El objetivo primordial de este artículo se suscribe en el interés por contribuir a la construcción de una propuesta epistemológica y metodológica que contemple las aportaciones de la arqueología, así como las de la antropología física y la etnografía, en los presupuestos de la antropología demográfica. Partimos de que un esfuerzo en el abordaje multidisciplinario que empiece desde la intradisciplina antropológica constituirá la aportación en torno a un objetivo común y primordial: la comprensión de las numerosas y variadas dinámicas de las complejas poblaciones humanas.

\section{Bibliografía}

Anderson, B. (1986). Regional and cultural factors in the decline of marital fertility in Europe. En A. Coale y S. Watkins (eds.). The decline of fertility in Europe (pp. 293-313). Princeton University Press.

Angel, J. L. (1969). The bases of paleodemography. American Journal of Physical Anthropology, 30(3), 427-437. Recuperado de https://online library.wiley.com/doi/abs/10.1002/ajpa.1330300314

Ardener, E. (1962). Divorce and fertility: An African study. Oxford: Oxford University Press for Nigerian Institute of Social and Economic Research. Arieta, V. (2013). Densidad poblacional olmeca y sus implicaciones en el sitio arqueológico San Lorenzo, Veracruz (Tesis de doctorado, Universidad Nacional Autónoma de México, Ciudad de México). Recuperado 
de http://www.ciencianueva.unam.mx/bitstream/handle/123456789 /156/26_densidad.pdf?sequence $=1 \&$ isAllowed $=y$

Arieta, V. y Cyphers, A. (2017a). Densidad poblacional en la capital olmeca de San Lorenzo, Veracruz. Ancient Mesoamerica, 28(1), 61-73. Recuperado de https://www.cambridge.org/core/services/aop-cambridgecore/content/view/CFC9D62298B5FB12A58BA5B53A96B248/ S0956536116000195a.pdf/densidad_poblacional_en_la_capital_olme ca_de_san_lorenzo_veracruz.pdf

Arieta, V. y Cyphers, A. (2017b). Etnografía, demografía y arqueología olmeca en San Lorenzo, Tenochtitlán. Revista Arqueología, 52, 7-26. Recuperado de https://www.researchgate.net/publication/321340206 Etnografia_demografia_y_arqueologia_olmeca_en_San_Lorenzo_Te nochtitlan

Arieta, V. y Cyphers, A. (s.f.). Dinámica y desarrollo de la población olmeca de San Lorenzo. Ciudad de México: Universidad Nacional Autónoma de México, Instituto de Investigaciones Antropológicas.

Aristóteles. (1953). Política, edición inglesa de 1932, Libro II. En Organización de las Naciones Unidas, Factores determinantes y consecuencias de las tendencias demográficas. Nueva York.

Axinn, W., Fricke, T. E. y Thornton, A. (1991). The microdemographic community-study approach: Improving survey data quality by integrating the ethnographic method. Sociological Methods and Research, 20(2), 187-217. Recuperado de http://journals.sagepub.com/doi/pdf/10.1177 /0049124191020002001

Balkansky, A. K., Kowalewski, S. A., Pérez Rodríguez, V., Pluckhahn, J. P., Smith, C. A., Stive, R. L., ... y Santos Pérez, R. (2000). Archaeological survey in the Mixteca Alta of Oaxaca, Mexico. Journal of Field Archaeology, 27(4), 365-389. Recuperado de https://www.tandfonline.com/doi/ abs/10.1179/jfa.2000.27.4.365

Barnes, J. A. (1949). Measures of divorce frequency in simple societies. The Journal of the Royal Anthropological Institute of Great Britain and Ireland, 79(1-2), 37-62. Recuperado de https://www.jstor.org/stable/ pdf/2844502.pdf

Bernardi, L. (2007). An introduction to anthropological demography (MPIDR Working Papers). Rostock, Alemania: Max Planck Institute for Demographic Research. Recuperado de https://www.researchgate. net/publication/4748211_An_introduction_to_anthropological_demog raphy

Binford, L. R., Binford, S. R., Whallon, R. y Hardin, M. A. (1970). Archaeology at Hatchery West. Memoirs of the Society for American Archaeol- 
ogy, 24, 1-91. Recuperado de https://www.jstor.org/stable/25146704? seq $=1$ \#metadata_info_tab_contents

Blanton, E. R. (1978). Monte Alban: Settlement patterns at the ancient Zapotec capital. Nueva York: Academic Press.

Blanton, E. R., Appel, J., Finsten, L., Kowalewski, S., Feinman, G. y Fisch, E. (1979). Regional evolution in the Valley of Oaxaca, Mexico. Journal of Field Archaeology, 6(4), 369-390. Recuperado de https://www.jstor. org/stable/529423?seq=1\#metadata_info_tab_contents

Bocquet-Appel, J. y Masset, C. (1982). Farewell to paleodemography. Journal of Human Evolution, 11(4), 321-333. Recuperado de https://www. sciencedirect.com/science/article/pii/S0047248482800237

Brown, M. (1987). Population estimation from floor area: A restudy of "Naroll's constant". Society for Cross-Cultural Research, 21(1-4), 1-49. Recuperado de http://journals.sagepub.com/doi/10.1177/106939718 702100101

Buikstra, J. E. y. Konigsberg, L. W. (1985). Paleodemography: Critiques and controversies. American Anthropologist New Series, 87(2), 316-333. Recuperado de https://www.jstor.org/stable/678564?seq=1\#metadata info tab contents

Caldwell, J. (1985). Strengths and limitations of the survey approach for measuring and understanding fertility change: Alternative possibilities. En J. Cleland y J. Hobcraft (eds.), Reproductive change in developing countries: Insights from the world fertility surveys (pp. 45-63). Londres: Oxford Univesity Press.

Caldwell, J., Caldwell, P. y Caldwell, B. (1987). Anthropology and demography. The mutual reinforcement of speculation and research. Current Anthropology, 28(1), 25-43. Recuperado de https://www.jstor.org/ stable/2743111?seq=1\#metadata_info_tab_contents

Caldwell, J., Hill, A. y Hull, V. (1988). Micro-approaches to demographic research. Londres: Kegan Paul International.

Chamberlain, A. (2006). Demography in archaeology. Cambridge: University Press.

Clarke, S. K. (1971). A method for the determination of Pre-Hispanic pueblo and population estimates. Prescott, AZ: Prescott College, Center for Man and the Environment.

Coale, A. J. y Watkins, S. C. (eds.). (1986). The decline of fertility in Europe. Princeton: Princeton University Press / Office of Population Research.

Coale, J. A. y Treadway, R. (1986). Summary of the changing distribution of overall fertility, marital fertility, and the proportion married in the provinces of Europe. En A. J. Coale y S. Cotts Watkins (eds.). The decline 
of fertility in Europe (pp. 31-181). Princeton: Princeton University Press / Office of Population Research.

Coe, M. D. y Diehl, R. (1980). In the land of the Olmec (2 vols.). Austin, TX: University of Texas Press.

Colson, E. (1958). Marriage and family among the plateau Tonga of Northern Rhodesia. Manchester: Manchester University Press.

Cook, E. (1972). Prehistoric demography. Reading, MA: Addison-Wesley. Cook Sherburne, F. y Heizer, F. R. (1968). Relationships among houses, settlement areas, and population in aboriginal California. En K. Chih Chang (ed.), Settlement archaeology (pp. 79-115). Palo Alto, CA: National Press Books.

Culbert, T. y Rice, D. (1990). Precolumbian population history in the Maya lowlands. Albuquerque, NM: University of New Mexico Press.

Cyphers, A. (1997). Población, subsistencia y medio ambiente en San Lorenzo Tenochtitlán. Ciudad de México: Universidad Nacional Autónoma de México, Instituto de Investigaciones Antropológicas.

Cyphers, A. (2012). Las bellas teorías y los terribles hechos. Controversias sobre los olmecas del preclásico inferior. Ciudad de México: Universidad Nacional Autónoma de México, Instituto de Investigaciones Antropológicas.

Cyphers, A. y Hirth, K. (2016). Transporte y producción artesanal en los albores del mundo olmeca. Ciudad de México: Universidad Nacional Autónoma de México, Instituto de Investigaciones Antropológicas.

Cyphers, A., Mutha, T., Borstein, J., Zurita, J., Lunagómez, R., Symonds, S., Jiménez, G., ... y Figueroa, J. M. (2007-2008). Arqueología digital en la primera capital olmeca, San Lorenzo. Thule, 22-25, 121-144.

Cyphers, A., Mutha, T., Zurita, J., Jiménez, G., Hernández, E., Martínez, B., Arieta, V., Lunagómez, R., Borstein, J., Symonds, S., Ortiz, M. A. y Figueroa, J. M. (2014). Atlas digital de la zona arqueológica de San Lorenzo, Veracruz. Ciudad de México: Universidad Nacional Autónoma de México.

Cyphers, A., Zurita, J. y Lane, M. (2013). Retos y riesgos en la vida olmeca. Ciudad de México: Universidad Nacional Autónoma de México, Instituto de Investigaciones Antropológicas.

D’ Aloja A. (1980). La población del Valle del Mezquital. Anales de Antropología, 17(1), 187-198.

D’ Aloja A. (1981). Fecundidad en un pueblo rural. Anales de Antropología, 18(1), 201-216. Recuperado de http://www.revistas.unam.mx/index.php/ antropologia/article/view/23944/pdf_827

D’ Aloja A. (1983). La hora del nacimiento en El Mezquital. Anales de An- 
tropología, 20(1) 147-156. Recuperado http://www.revistas.unam.mx/ index.php/antropologia/article/view/415/395

D' Aloja A. (1997). Mortalidad infantil en una zona rural del Valle del Mezquital. Estudios de Antropología Biológica, 6, 119-127. Recuperado de http://www.revistas.unam.mx/index.php/eab/article/view/42726/38817

Daneels, A. (1997). Settlement history in the lower Cotaxtla drainage. En B. Stark y P. J. Arnold (eds.), Olmec to Aztec: Settlement patterns in the ancient gulf coast lowlands (pp. 206-252). Tucson, AZ: University of Arizona Press.

Domínguez Carrasco, M. y Folan, W. J. (1999). La cerámica de Calakmul, Campeche. Primeros resultados. Information, 16, 29-48.

Fortes, M. (1943). A note on fertility among the Tallensi of the Gold Coast. The Sociological Review, 35(4-5), 99-113. Recuperado de http://journals. sagepub.com/doi/abs/10.1111/j.1467-954X.1943.tb02246.x

Harris, M. (1986). Caníbales y reyes. Barcelona: Salvat.

Hassan, F. (1978). Demographic archaeology. Advances in Archaeological Method and Theory, 1, 49-103. Recuperado de https://www.science direct.com/science/article/pii/B9780120031016500093

Hassan, F. (1981). Demographic archaeology. Washington, DC: Washington State University, Department of Anthropology / Academic Press.

Haviland, W. (1969). A new population estimate for Tikal, Guatemala. American Antiquity, 34(4), 429-433. Recuperado de https://www.jstor.org/ stable/277741?seq=1\#metadata_info_tab_contents

Hernández Espinoza, P. (2006). La regulación del crecimiento de la población en el México prehispánico. Ciudad de México: Instituto Nacional de Antropología e Historia.

Hernández Espinoza, P. (2010). La antropología demográfica: una propuesta desde la antropología física. Ponencia presentada en el I Congreso Nacional de Antropología Social y Etnología “Globalización, Diversidad y Práctica Antropología”. Ciudad de México: Colegio de Etnólogos y Antropología Social A.C. (CEAS) / Universidad Autónoma Metropolitana.

Hernández Espinoza, P. y Márquez Morfín, L. (2003). La demografía en la antropología: una propuesta metodológica. Estudios de Antropología Biológica, 11(2), 693-721. Recuperado de http://www.revistas.unam. mx/index.php/eab/article/view/43206/39221

Hernández Guevara, E. (2000). Las investigaciones de los montículos bajos en San Lorenzo: consideraciones sobre estimaciones poblacionales olmecas (Tesis de Licenciatura en Arqueología). Xalapa. Ver.: Universidad Veracruzana. 
Hoppa, R. (2002). Paleodemography: Looking back and thinking ahead. En R. D. Hoppa y J. W. Vaupel (eds.), Paleodemography: Age distributions from skeletal samples. Cambridge studies in biological and evolutionary anthropology (pp. 9-28). Nueva York: Cambridge University Press.

Jochim, M. A. (1976). Hunter-gatherer subsistence and settlement: A predictive model. Nueva York: Academic Press.

Kardulias, P. N. (1992). Estimating population at ancient military sites: The use of historical and contemporary analogy. American Antiquity, 57(2), 276-287. Recuperado de https://www.jstor.org/stable/280733?seq=1\# metadata_info_tab_contents

Keene, A. S. (1977). Economic optimization models and the study of hundergatherer subsistence settlement systems. Documento presentado en la 42nd Annual Meeting of the Society for American Archaeology, Nueva Orleans, 30 de abril.

Kertzer, D. y Fricke, T. (eds.). (1997). Anthropological demography. Chicago, ILL.: The University of Chicago Press.

Kolb, C. (1985). Demographic estimates in archaeology: Contributions from ethnoarchaeology on Mesoamerican peasants. Current Anthropology, 26(5), 581-599. Recuperado de https://www.jstor.org/stable/2743081? seq=1\#metadata_info_tab_contents

Kowalewski, S. A., Feinman, G., Finsten, L., Blanton, R. E. y Nicholas, L. M. (1989). Monte Albán's hinterland, Part II: Prehispanic settlement patterns in Tlacolula, Etla, and Ocotlán, the Valley of Oaxaca, Mexico. Ann Arbor, MI: University of Michigan (Memoirs, Museum of Anthropology).

Laporte, J. P. y Torres, C. R. (1988). Proyecto Sureste de Petén, segunda temporada, 1987-1988. Reconocimientos Arqueológicos en el Valle de Dolores. Atlas Arqueológico de Guatemala. Ciudad de Guatemala.

Leasure, J. W. (1963). Factors involved in the decline of fertility in Spain, 1900-1950. Population Studies, 16(3), 271-285. Recuperado de https:// www.jstor.org/stable/2172782?seq=1\#metadata_info_tab_contents

LeBlanc, S. (1971). An addition to Naroll's suggested floor area and settlement population relationship. American Antiquity, 36(2), 210-211. Recuperado de https://www.cambridge.org/core/journals/americanantiquity/article/an-addition-to-narolls-suggested-floor-area-and-settle ment-population-relationship/FF461CC0F912CD9E0C494EC2804B 25FD

Leshaeghe, R. y Surkyn, J. (1988). Cultural dynamics and economic theories of fertility change. Population and Development Review, 14(1), 1-45. Recuperado de https://www.jstor.org/stable/1972499?seq=1\#metadata info tab contents 
Malinowski, B. (1922). Argonauts of the Western Pacific. Londres: Routledge \& Kegal Paul.

Malthus, T. (1798). An essay on the principle of population. Londres: J. Johnson.

Mann, A. (1975). Some paleodemographic aspects of the South African Australopithecines. Filadelfia, PA: University of Pennsylvania.

McKinley, K. (1971). Survivorship in gracile and robust Australopithecines: A demographic comparison and a proposed birth model. American Journal of Physical Anthropology, 34(3), 417-426. Recuperado https:// onlinelibrary.wiley.com/doi/pdf/10.1002/ajpa.1330340311

Millennium Ecosystem Assessment (MEA). (2005). Ecosystems and human well-being: Synthesis report. Washington, DC: Island Press.

Mitchell, J. C. (1949). An estimate of fertility in some Yao hamlets in Liwonde district of Southern Nyasaland. Africa: Journal of the International African Institute, 19(4), 293-308. Recuperado de https://www.jstor.org/ stable/1156405?seq=1\#metadata_info_tab_contents

Naroll, R. (1962). Floor area and settlement population. American Antiquity, 27(4), 587-589. Recuperado de https://www.cambridge.org/core/jour nals/american-antiquity/article/div-classtitlefloor-area-and-settlement-po pulationdiv/85BCCD6D2BCA38359771F474410F5802

Nelson, Z. (2004). De la cartografía al cálculo de población de Piedras Negras, Guatemala. En J. P. Laporte, B. Arroyo, H. Escobedo y H. Mejía (eds.). XVII Simposio de Investigaciones Arqueológicas en Guatemala, 2003. Guatemala: Museo Nacional de Antropología y Etnología.

Oliver, A. y Gusi, F. (1995). El Puig de la Nau. Un hábitad fortificado ibérico en el ámbito mediterráneo peninsular (Monografies de Prehistória i Arqueologia Castellonenques, 4). Castellón, España: Diputació de Castelló.

Orcutt, J. D. (1974). The measurement of prehistory population size: The Chevelon region of Arizona (Tesis de maestría, University of California, Los Ángeles).

Ortega, A. (2004). La paleodemografía: ¿un instrumento para simular el comportamiento demográfico del pasado? Análisis comparativo con la demografía histórica en la Ciudad de México del siglo XIX. Estudios Demográfico y Urbanos, 19(1), 181-214. Recuperado de https://estu diosdemograficosyurbanos.colmex.mx/index.php/edu/article/view/1199 $/ 1192$

Ortiz, M. A. y Cyphers, A. (1997). La geomorfología y las evidencias arqueológicas en la región de San Lorenzo Tenochtitlán, Veracruz. En A. Cyphers (coord.). Población, subsistencia y medio ambiente en San 
Lorenzo. Ciudad de México: Universidad Nacional Autónoma de México, Instituto de Investigaciones Antropológicas.

Ortman, S., Cabaniss, A., Sturm, J. y Bettencourt, L. (2014). The pre-history of urban scaling. Plos One, 9(2), 1-10. Recuperado de https://journals. plos.org/plosone/article/file?id=10.1371/journal.pone. $0087902 \&$ type $=$ printable

Parsons, J. R. (1968). An estimate of size and population for middle horizon Tiahuanaco, Bolivia. American Antiquity, 33(2), 243-245. Recuperado de https://www.jstor.org/stable/278529?seq=1\#metadata_info_tab_con tents

Parsons, R. J. (1989). Arqueología regional en la Cuenca de México: una estrategia para la investigación futura. Anales de antropología, 26(1), 157-257. Recuperado de http://www.revistas.unam.mx/index.php/ antropologia/article/view/13033/12349

Platón. (1999). Diálogos. Obra completa. Volumen V-VIII: Leyes (Libro I-IV). Introducción, traducción y notas de Francisco Liso. Madrid: Gredos.

Pringle, D. (1988). The defense of Byzantine Africa from Justinian to the Arab conquest. Oxford: British Archaeological Records.

Puleston, D. (1973). Ancient Maya settlement patterns and environment at Tikal, Guatemala: Implications for subsistence models (Tesis de doctorado, Filadelfia, University of Pennsylvania).

Redfield, R. y Villa Rojas, A. (1934). Chan Kom: A Maya village. Washington, DC: Carnegie Institution of Washington.

Richards, I. A. (1940). Bemba marriage and present economic conditions. Papers of the Rhodes- Livingstone Institute, 4.

Royal Anthropological Institute of Great Britain and Ireland. (1951). Notes and queries on Anthropology. Londres: Routledge and K. Paul.

Sahlins, M. (1974). Economía de la Edad de Piedra. Madrid: Akal.

Sanders, W. (1962-1963). Cultural ecology of the Maya lowlands. Estudios de Cultura Maya, 2, 79-121. Recuperado de https://revistas-filologicas. unam.mx/estudios-cultura-maya/index.php/ecm/article/view/215/216

Sanders, W., Parsons, R. J. y Santley, S. R. (1979). The basin of Mexico: Ecological processes in the evolution of a civilization. Nueva York: Academic Press.

Sandey, S. R. y Arnold, P. J. (1996). Prehispanic settlement patterns in the Tuxtla Mountains, southern Veracruz, Mexico. Journal of Field Archaeology, 23(2), 225-249. Recuperado de https://www.tandfonline.com/doi/ abs/10.1179/009346996791973963

Santley, S. R., Arnold III, P. J. y Barrett, P. T. (1997). Formative period 
settlement patterns in the Tuxtla Mountains. En B. Stark y P. Arnold III (eds.), Olmec to Aztec, settlement patterns in the ancient gulf lowlands (pp.174-205). Tucson: University of Arizona Press.

Schapera, I. (1955). An anthropologist's approach to population growth: Studies in the Bechuanaland Protectorate. En J. B. Cragg y N. W. Pirie (eds.), The numbers of man and animals (pp. 23-29). Edimburgo: Oliver and Boyd.

Soudsky, B. (1969). Étude de la maison néolithique. Slovenská Archeólogic 17(1), 5-96.

Stark, B. L. y Curet, L. A. (1994). The development of the classic-period Mixtequilla in south-central Veracruz, Mexico. Ancient Mesoamerica, 5(2), 267-287. Recuperado de https://www.jstor.org/stable/26307 302?seq=1\#page_scan_tab_contents

Swedlund, A. C. y Armelagos G. J. (1976). Demographic anthropology. Dubuque, IA: WMC Brown Company Publishers.

Symonds, S., Cyphers, A. y Lunagómez, R. (2002). Asentamiento prehispánico en San Lorenzo, Tenochtitlán. Ciudad de México: Universidad Nacional Autónoma de México.

Wilk, R. y Ashmore, W. (1988). Household and community in the Mesoamerican past. Albuquerque, NM: University of New Mexico Press.

Wilmsen, E. N. (1973). Interaction, spacing behavior and the organization of hunting bands. Journal of Anthropological Research, 29(1), 1-31. Recuperado de https://www.jstor.org/stable/3629622?seq=1\#metadata info_tab_contents

Wobst, H. M. (1975). The demography of finite populations and the origins of the incest taboo. Memoirs of the Society for American Archaeology, 30, 75-81. Recuperado de https://www.jstor.org/stable/25146735? seq=1\#metadata_info_tab_contents

Zubrow Ezra, B. W. (1976). Demographic anthropology: Quantitative approaches, Albuquerque: University of New Mexico Press.

Zurita, J. (1997). Los fitolitos: indicaciones sobre dieta y vivienda en San Lorenzo. En A. Cyphers (coord.), Población, subsistencia y medio ambiente en San Lorenzo Tenochtitlán (pp. 75-87). Ciudad de México: Universidad Nacional Autónoma de México, Instituto de Investigaciones Antropológica. 


\section{Acerca de la autora}

Virginia Arieta Baizabal es doctora en Antropología por la Universidad Nacional Autónoma de México (UNAM), maestra en Estudios Mesoamericanos por la misma institución, y arqueóloga por la Universidad Veracruzana (UV). Es investigadora del Instituto de Antropología (UV) y docente en los programas de licenciatura y maestría de la Facultad de Antropología (UV). Es integrante activa del Consejo Internacional de Monumentos y Sitios de la UNESCO (Icomos mexicano), así como miembro del Sistema Nacional de Investigadores (SNI), de la Sociedad Mexicana de Demografía (Somede) y de la Sociedad Americana de Arqueología (SAA). De 2005 a 2016 colaboró en el Proyecto Arqueológico San Lorenzo Tenochtitlán (UNAM), en donde desarrolló una investigación sobre densidad y cálculo poblacional en el sitio olmeca. Actualmente dirige el proyecto de investigación arqueológica "Dinámica poblacional e interacciones sociopolíticas en la región de Capoacan, Veracruz. Proyecto Arqueológico Antonio Plaza", del Instituto de Antropología (UV). Sus principales temas de interés incluyen: cultura olmeca, sistemas de información geográfica, cálculos poblacionales, arqueología demográfica y dinámicas de la población prehispánica. Es autora de libros, capítulos de libros, artículos científicos y de divulgación. Es directora editorial de Fuimos Peces, revista digital de ciencias sociales y humanidades. Consúltese: https:// uv-mx.academia.edu/VirginiaArieta ORCID: https://orcid.org/0000-00020932-6272

Recepción: 13 de marzo de 2018.

Aceptación: 18 de septiembre de 2018. 